\title{
Survival impact of delaying postoperative chemoradiotherapy in newly-diagnosed glioblastoma patients
}

\author{
Maochen Zhang, Fei Xu, Weiqiong Ni, Weixiang Qi, Weiguo Cao, Cheng Xu, Jiayi Chen, Yunsheng Gao \\ Department of Radiation Oncology, Ruijin Hospital, Shanghai Jiao Tong University, School of Medicine, Shanghai, China \\ Contributions: (I) Conception and design: M Zhang, F Xu, Y Gao; (II) Administrative support: W Cao, C Xu, J Chen; (III) Provision of study \\ materials or patients: M Zhang, Y Gao; (IV) Collection and assembly of data: M Zhang, F Xu, W Ni; (V) Data analysis and interpretation: M Zhang, \\ W Qi, Y Gao; (VI) Manuscript writing: All authors; (VII) Final approval of manuscript: All authors. \\ Correspondence to: Yunsheng Gao, MD. Department of Radiation Oncology, Ruijin Hospital, Shanghai Jiao Tong University, School of Medicine, 197 \\ Ruijin Second Road, Shanghai 200025, China. Email: gys11856@rjh.com.cn.
}

\begin{abstract}
Background: Maximal safe resection followed by adjuvant chemoradiotherapy (CRT) with temozolomide (TMZ) is the standard treatment for newly diagnosed glioblastoma multiforme (GBM) patients. Time of initiation of postoperative adjuvant therapy has been demonstrated to impact on prognosis. For GBM patients, the optimal interval between definitive surgery and CRT is still uncertain. Current study aims to find whether the delayed initiation of CRT after surgery has a negative impact on patients' outcome.

Methods: Sixty-six consecutively patients with newly-diagnosed GBM treated with surgery and adjuvant CRT from April 2014 to September 2019 at Ruijin Hospital School of Medicine Shanghai Jiaotong University were retrospectively reviewed. The impact of postoperative time from surgery to adjuvant treatment on patient's overall survival (OS) and progression-free survival (PFS) were evaluated by univariate Log-rank test and multivariate Cox regression analysis. Factors including age, Karnofsky performance status (KPS), maximum diameter of primary tumor, extent of resection, isocitrate dehydrogenase (IDH) mutation status and O6-methylguanine-DNA-methyltransferase (MGMT) promoter methylation status were also analyzed in Cox regression model.
\end{abstract}

Results: The median OS for patients who started CRT less than 6 weeks $(n=48)$ and more than 6 weeks $(\mathrm{n}=18)$ were 26.6 months (95\% CI: 18.3-34.9) and 15.7 months (95\% CI: 9.2-22.3) (P=0.001). The median PFS for the short interval group was 16.3 months (95\% CI: 14.7-18.0) and for the long interval group was 9.1 months (95\% CI: 4.7-13.4) ( $\mathrm{P}=0.006)$. On multivariate Cox regression analysis, high KPS and initiation of CRT less than 6 weeks were two independent prognostic factors for better OS and PFS (all $\mathrm{P}<0.05$ ).

Conclusions: Initiation of adjuvant CRT beyond 6 weeks contributed to worse survival in GBM patients, therefore CRT should be initiated within 6 weeks after surgery.

Keywords: Glioblastoma; radiotherapy; delay; survival; prognostic factors

Submitted Mar 29, 2020. Accepted for publication Aug 10, 2020.

doi: $10.21037 /$ tcr-20-1718

View this article at: http://dx.doi.org/10.21037/tcr-20-1718

\section{Introduction}

Glioblastoma multiforme (GBM) is the most common malignant brain tumor in adults. In 2005, the research by Stupp and his colleagues brought significant advances in treatment. Ever since then, maximum safe resection followed by adjuvant chemoradiotherapy (CRT) with temozolomide (TMZ) is the standard therapy for GBM (1).
However, the survival time of GBM is still unsatisfactory, which rarely exceeds 14 months $(2,3)$.

Extensive studies have focused on the effort to optimize the delivery of radiotherapy (RT), among which includes the waiting time of initiation from surgery to adjuvant CRT. Evidence of higher recurrence rates and worse outcomes due to delayed initiation of postoperative treatment has 
already been demonstrated in other types of malignant tumors such as breast (4-6), head and neck $(6,7)$ and lung cancer $(7,8)$. From the radiobiological point of view, the efficacy of RT decreases as the tumor size increases, especially for highly proliferative tumors with rapid growth such as GBM (9-13). It is suggested that longer delay between surgery and postoperative treatment is a detrimental factor for early tumor regrowth and associated with poorer prognosis (14-16).

Up to now, the optimal interval between surgery and CRT in GBM is still an open question and whether the delay of initiation of CRT has adverse effect on patient's survival outcomes remains controversial. In some studies, the expected detrimental impact of delayed initiation of CRT was identified while others failed to find any relationship between interval time and prognosis. Some research even showed a beneficial effect of moderately delayed RT on prognosis.

The aim of our study was to evaluate the impact of delayed initiation of CRT on survival in newly diagnosed GBM patients, also taking other prognostic factors such as age, Karnofsky performance status (KPS), maximum diameter of primary tumor, extent of resection, isocitrate dehydrogenase (IDH) mutation status and O6-methylguanine-DNA-methyltransferase (MGMT) promoter methylation status into account (17-21).

We present the following article in accordance with the STROBE reporting checklist (available at http://dx.doi. org/10.21037/tcr-20-1718).

\section{Methods}

\section{Study design and eligibility}

We retrospectively analyzed all patients with newly diagnosed GBM at Ruijin Hospital School of Medicine Shanghai Jiaotong University from April 2014 to September 2019. All the enrolled patients underwent surgical resection followed by the standard CRT and were histologically proven GBM according to the 2016 WHO Classification of Tumors of the Central Nervous System (CNCS). Patients with unknown date of surgery or initiation of adjuvant CRT were excluded. A total of 66 patients were included.

\section{Patients and pretreatment evaluation}

Electronic medical records were reviewed for patient characteristics such as age, gender, KPS, maximum diameter of primary tumor, extent of resection and the interval between surgery and CRT. Data of tumor molecular markers including IDH mutation status and MGMT promoter methylation status were also collected. All information was anonymized. The study was conducted in accordance with the Declaration of Helsinki (as revised in 2013). This study was approved by the Ethics Committee of Ruijin Hospital affiliated to Shanghai Jiao Tong University School of Medicine (ID: 2020-192), and written informed consent was not required because of the retrospective nature of the study.

\section{Treatment planning and delivery}

All patients received radiation therapy (RT) and were immobilized in the supine position with a tailored headthermoplastic mask, followed by a CT simulation at a slice thickness of $3-5 \mathrm{~mm}$. CT scans were fused with contrast-enhanced MRI imaging within two weeks before radiotherapy, and delineated slice-by-slice according to EORTC or RTOG guidelines. The standard prescription dose is 60 Gy (2 Gy per day from Monday to Friday). A 6-MV photon beam is provided by a Varian TrueBeam or Elekta linear accelerator using a multi-leaf collimator.

\section{Combined chemotherapy}

All the enrolled patients received concurrent CRT with TMZ. The TMZ regimen was continuous daily dosage of $75 \mathrm{mg} / \mathrm{m}^{2} /$ day for 7 days a week during the whole RT period, and was followed by 6 cycles of adjuvant TMZ (150 to $200 \mathrm{mg} / \mathrm{m}^{2}$ for 5 days every 28 days).

\section{Follow-up}

Follow-up including a routine contrast-enhanced MRI to the head was performed every 3 months or upon clinical deterioration.

\section{Statistical analysis}

Continuous variables were described using median and range while categorical variables by frequency and percentage. Overall survival (OS) was defined as time from surgery until death. Progression-free survival (PFS) was defined as time from surgery until the diagnosis of progression based on imaging or death.

The interval between surgery and CRT were classified 
into two groups: initiation of CRT $\leq 6$ weeks and initiation of CRT $>6$ weeks. Data from the two groups were compared by using Chi-square test for categorical variables and two-sample $t$-test for continuous variables. $\mathrm{P}$ values of less than 0.05 were considered significant. Kaplan-Meier survival curves were constructed between the two CRT interval groups using Log-rank test in univariate analysis. Well-recognized prognostic factors including age, KPS, maximum diameter of primary tumor, extent of resection, IDH status and MGMT status as well as interval between surgery and CRT were measured using univariate Cox regression analysis. Time interval and other prognostic factors with a $\mathrm{P}$ value less than 0.1 in univariate analysis were then included in the multivariate Cox regression model. Statistical analyses were conducted using SPSS 21.0 (IBM Corporation, Armonk, NY, USA).

\section{Results}

Patient characteristics were summarized in Table 1. Among the 66 enrolled patients, $45(68.2 \%)$ were male and 21 (31.8\%) were female, with a mean age of $55.7 \pm 12.8$ years and a median KPS of 80 (range, 50-90). The median interval of initiation of CRT $\leq 6$ weeks group was 4.4 (range, 1.4-6) weeks and 7.2 (range, 6.1-23.7) weeks for the initiation of CRT $>6$ weeks group. No difference was observed between the short interval and long interval group in terms of gender, age, KPS, preoperative tumor size, extent of resection, IDH status and MGMT status.

The median OS and PFS for all patients in our study were 21.6 months (95\% CI: $17.3-25.8$ ) and 16.0 months (95\% CI: 14.1-17.8). The 1-, 2- and 3-year survival rates were $88.9 \%, 37.2 \%$ and $14.0 \%$, respectively. Median OS for the short interval ( $\leq 6$ weeks) group was 26.6 months $(95 \%$ CI: $18.3-34.9)$ and for the long interval ( $>6$ weeks) group 15.7 months (95\% CI: 9.2-22.3) $(\mathrm{P}=0.001)$ (Figure 1). For PFS, the short interval group was 16.3 months (95\% CI: 14.7-18.0) compared to the long interval group 9.1 months (95\% CI: 4.7-13.4) $(\mathrm{P}=0.006)$ (Figure 2).

The univariate Cox regression analysis of wellrecognized prognostic factor's influence on OS and PFS were listed in Table 2 and Table 3. Initiation of CRT less than 6 weeks suggested longer OS (HR: 4.34, 95\% CI: 1.66-11.33, P=0.003) and PFS (HR: 3.16, 95\% CI: $1.33-$ 7.49, $\mathrm{P}=0.009)$. There was a trend high KPS $(\mathrm{P}=0.060)$ associated with better OS. For PFS, high KPS $(\mathrm{P}=0.008)$ was identified as a beneficial factor.

In multivariate Cox regression analysis, we included all the variables from univariate analysis with a $\mathrm{P}$ value lower than 0.1. High KPS and initiation of CRT less than 6 weeks were independently associated with longer OS and PFS (all $\mathrm{P}<0.05)$ (Table 2 and Table 3).

\section{Discussion}

This study was aimed to evaluate the impact of delayed initiation of CRT on survival outcomes in GBM patients. In our study, all the patients received a standard RT dose of $60 \mathrm{~Gy}$ with concurrent TMZ. Our results indicated that initiation of CRT within 6 weeks was an independent prognostic factor for longer OS and PFS.

We chose the cut-off point of 6 weeks since this was the longest delay permitted in most GBM clinical trials (22). Radiation oncologists are aware of a potential negative impact of delays and thus avoid longer time intervals (23). There was a tendency to begin postoperative RT within this timeframe of $4-6$ weeks $(22,24)$. Within this period, most research did not find impact of delayed initiation of CRT on survival outcomes. However, prolonged delay beyond 6 weeks seemed to have a different result (25). In some areas, a waiting time between surgery and CRT longer than 6 weeks is not uncommon due to lack of RT facilities and patient's poor illness perceptions (26). Our study suggested this prolongation could lead to a worse outcome.

Mathematical modelling showed GBM is a highly proliferative tumor with a doubling time of 24 days (10). Late initiation of RT could lead to reduced radiosensitivity. De Barros et al. compared preoperative, immediate postoperative and preradiotherapy MRI images of 75 patients, among which $72 \%$ had an early tumor regrowth (14). Patients with no early tumor growth had a better OS than those with early tumor regrowth by 6.9 months. Villanueva-Meyer et al. also observed as many as $48 \%$ patients had new or increased contrast enhancement on MRI images during the waiting time of RT (15). Both OS and PFS were shorter in those who developed early tumor growth. The authors supposed slight increase in early tumor progression rates within 3-6 weeks while progression would increase in a significant manner with longer delays beyond 6 weeks.

In line with our data, several reports demonstrated delaying RT as a detrimental factor for survival. In a single institutional research, Do et al. found the risk of death increased by $2 \%$ for each day of waiting for RT in high-grade glioma patients (27). In Irwin et al.'s analysis of 172 patients, every additional week of delay until the 
Table 1 Clinical characteristics of the 66 glioblastoma patients according to interval between surgery and CRT

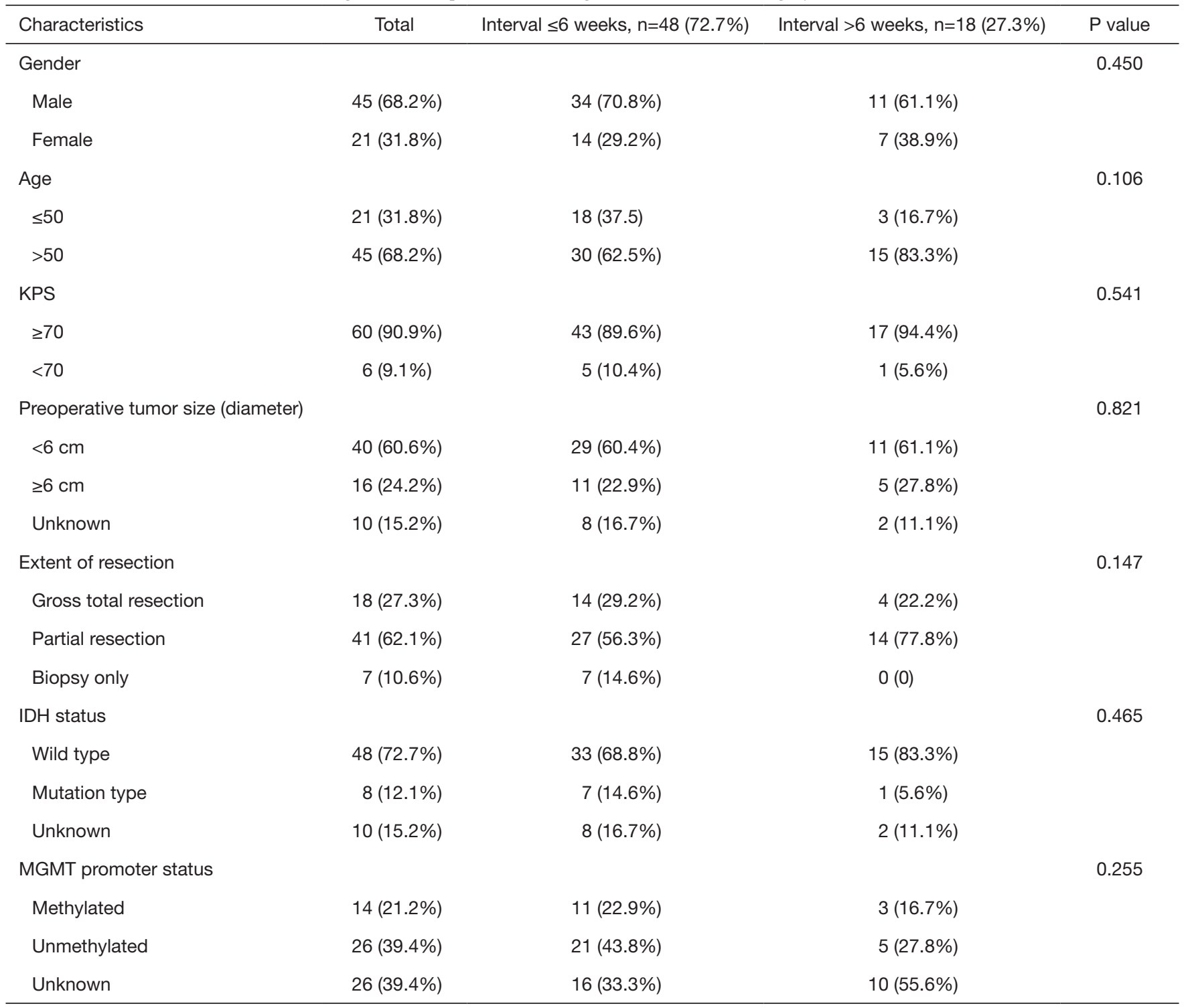

CRT, chemoradiotherapy; KPS, Karnofsky performance status; IDH, isocitrate dehydrogenase; MGMT, O6-methylguanine-DNAmethyltransferase.

start of RT increased the risk of death by $8.9 \%$ and a 6 week delay in starting RT reduced median survival by 11 weeks (28). However, the major limitation of these two studies was the inclusion of both grade III and grade IV glioma patients, which was recognized to be with different behaviors and survival outcomes. Another limitation was the heterogeneous RT doses they delivered, which was also known correlated with treatment efficacy. In our study, only GBM patients were included and all the patients received a total RT dose of 60 Gy with the same RT technique.
Valduvieco et al.'s study also showed a longer median OS of 21.3 months in patients who started RT within 6 weeks compared to those who initiated beyond 6 weeks with only 14.1 months (22). Patients enrolled in this study all underwent complete resection, which might restrict its generalizability. Another limitation should be pointed out was that TMZ was not used in all patients in this study. Spratt $e t a l$. also suggested a negative effect of delaying RT longer than 6 weeks in a cohort with known MGMT status information (29). Sun et al. demonstrated initiation 


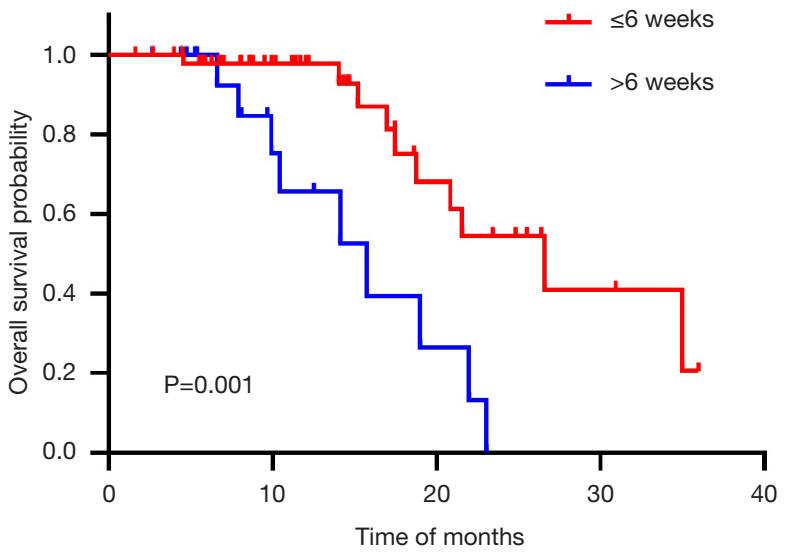

Figure 1 Kaplan-Meier overall survival of patients with initiation of CRT $\leq 6$ weeks and $>6$ weeks. CRT, chemoradiotherapy.

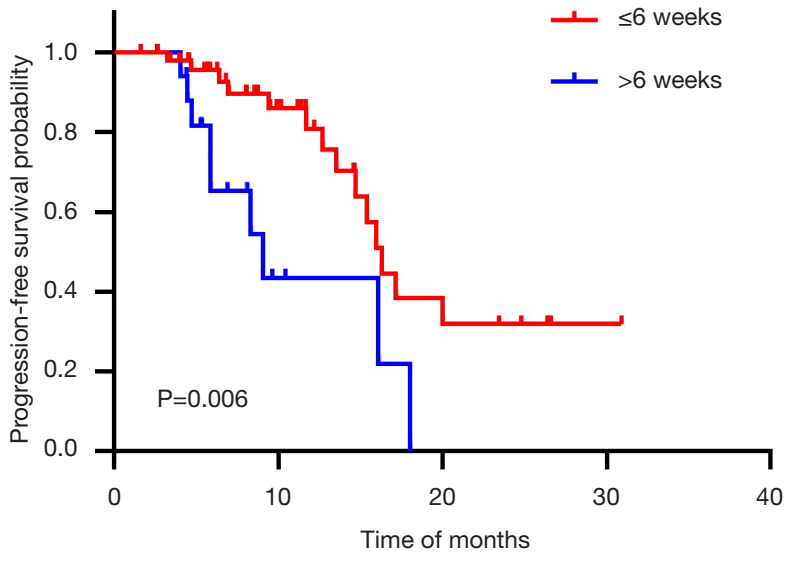

Figure 2 Kaplan-Meier progression-free survival of patients with initiation of CRT $\leq 6$ weeks and $>6$ weeks. CRT, chemoradiotherapy.

Table 2 Univariate and multivariate predictors of overall survival

\begin{tabular}{|c|c|c|c|c|c|c|c|}
\hline Variables & Subtype & \multicolumn{3}{|c|}{ Univariate analysis } & \multicolumn{3}{|c|}{ Multivariate analysis } \\
\hline \multirow[t]{2}{*}{ Age } & $\leq 50$ & 1 (ref) & & & & & \\
\hline & $>50$ & 0.60 & $0.22-1.62$ & 0.312 & & & \\
\hline KPS & $\geq 70$ & 1 (ref) & & & 1 (ref) & & \\
\hline \multirow[t]{2}{*}{ Preoperative tumor size (diameter) } & $<6 \mathrm{~cm}$ & 1 (ref) & & & & & \\
\hline & $\geq 6 \mathrm{~cm}$ & 1.61 & $0.59-4.40$ & 0.357 & & & \\
\hline \multirow[t]{2}{*}{ Extent of resection } & Gross total resection & 1 (ref) & & & & & \\
\hline & Partial resection & 0.68 & $0.19-2.43$ & 0.553 & & & \\
\hline IDH status & Wild type & 36.71 & - & 0.275 & & & \\
\hline \multirow[t]{2}{*}{ MGMT promoter status } & Methylated & 1 (ref) & & & & & \\
\hline & Unmethylated & 41.35 & - & 0.668 & & & \\
\hline \multirow[t]{2}{*}{ Interval time between surgery and CRT } & $\leq 6$ weeks & 1 (ref) & & & 1 (ref) & & \\
\hline & $>6$ weeks & 4.34 & $1.66-11.33$ & 0.003 & 6.64 & 2.20-20.04 & 0.001 \\
\hline
\end{tabular}

CRT, chemoradiotherapy; KPS, Karnofsky performance status; IDH, isocitrate dehydrogenase; MGMT, O6-methylguanine-DNAmethyltransferase; ref, reference.

of adjuvant RT beyond 6 weeks had a significant worsened impact on OS. But one restriction should be raised in this study was that some important confounding factors known to impact outcomes such as KPS and extent of resection were not contained (25). Pollom et al. and Graus et al. declared the benefits in survival obtained with optimal surgical resection may be reduced by prolonged initiation of RT $(26,30)$.

However, contradictory findings were reported in multiple other research. A meta-analysis including 12 
Table 3 Univariate and multivariate predictors of progression-free survival

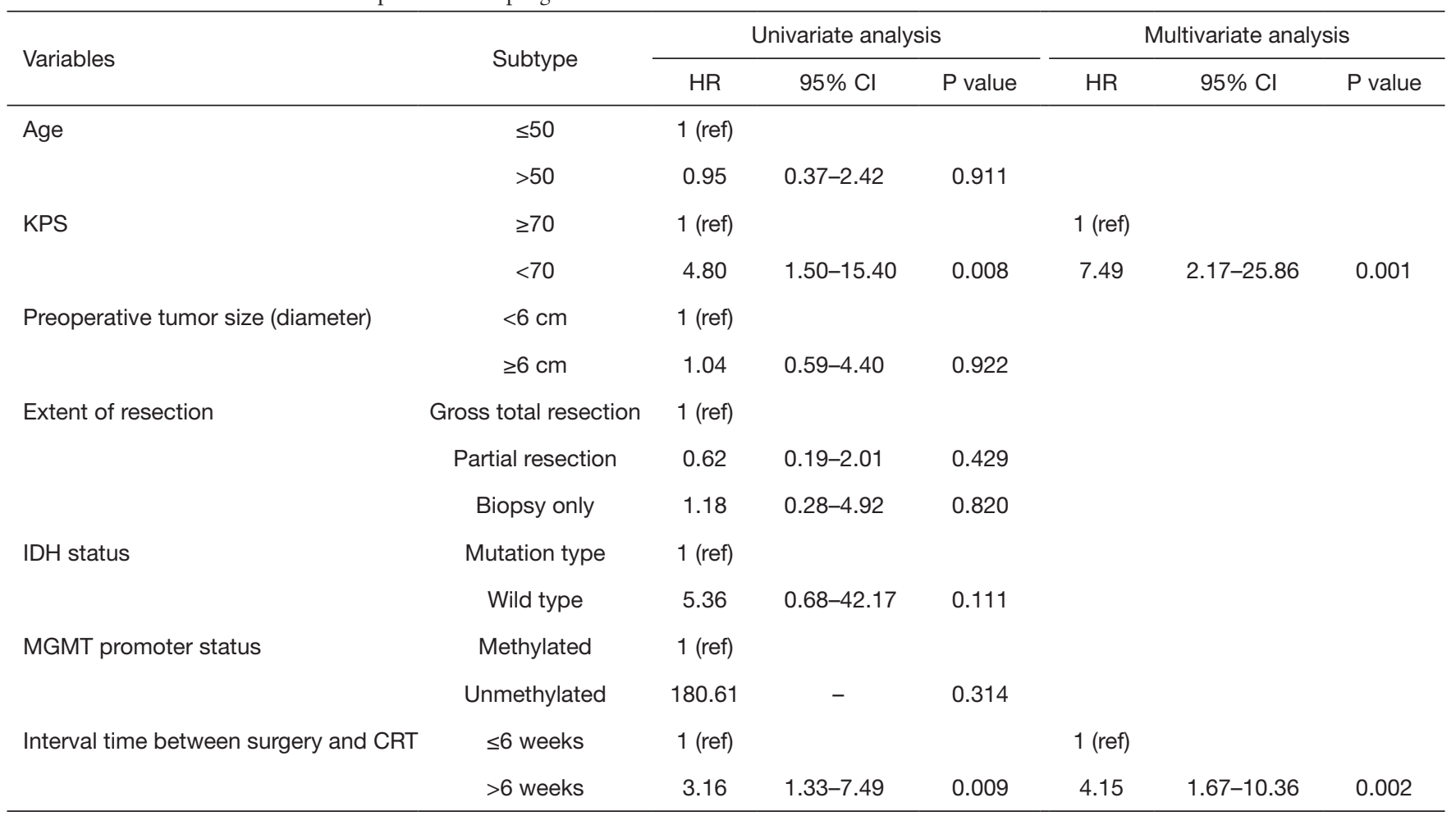

CRT, chemoradiotherapy; KPS, Karnofsky performance status; IDH, isocitrate dehydrogenase; MGMT, O6-methylguanine-DNAmethyltransferase; ref, reference.

studies of 5,212 patients found no evidence of reduced treatment outcomes caused by delayed initiation of RT (31). In this meta-analysis, one single retrospective analysis of 16 RTOG randomized trails comprised 2,855 patients, almost half of the cohort. Besides, the initiation of RT in this study was restricted to within 6 weeks as an eligibility criterion. In order to eliminate the bias caused by the high weight $(26 \%)$ of this study and time period restrictions, the authors excluded those studies with limitations and reanalyzed the data, but received similar main results. It was interesting to note that this RTOG study even suggested a better survival for patients who started RT longer than 4 weeks compared to those who began RT within 2 weeks after surgery $(32,33)$. These unexpected findings might possibly be explained by that postoperative RT is initiated more immediately for patients with poor prognostic characteristics such as low KPS, old age and undergoing biopsy only (30,32-34). In early postoperative period, hypoxia and edema surrounding the surgical bed could induce radioresistance. Besides, expanded irradiated volume because of insufficient shrinking of surgical cavity would lead to increased damage to normal brain tissue, associated with a worse clinical status and poor survival $(32,35)$. Animal experiments on rats also indicated that early irradiation after surgery induced greater brain injury (36). Another study based on the Clinformatics Data Mart database also supported early initiation of postoperative RT for high-grade glioma was not associated with increased survival (24). Similar to the RTOG study, starting RT within 4 weeks after surgery even demonstrated a worse survival compared to initiation of $4-13$ weeks later, but not significant in multivariate analysis. In a singlecenter analysis of pediatric population, Azizi et al. identified interval from surgery to RT not a significant risk factor (37). Only 38 children were included in the study and the cutoff point of RT initiation was 17 days, with only 9 children starting RT beyond 28 days, much shorter than the published data in adult cohorts. Thomson et al., Lai et al. and Noel et al. also failed to find any relationship between delaying RT and survival rates for GBM patients (38-40).

In the era of TMZ, its significant additive effects in combination with RT might render time initiation of RT as a less important predictor of survival outcomes. Whether 
RT could be delayed in GBM patients still remained a question difficult to answer. It seemed to be no detriment when delaying standard CRT within 6 weeks time-frame. But prolonged delay beyond 6 weeks should be avoided probably.

There were several limitations in our study. First of all, disadvantages such as selection and clinical data bias were hard to avoid due to the retrospective nature of our study. Prospective randomized clinical trials are still needed to prove the effect of initiation of postoperative CRT on survival, although ethical concerns might not permit. Secondly, the sample of our cohort is small, and the information of molecular markers including IDH status and MGMT promoter methylation status was not available for all patients. Prognostic factors such as extent of resection and molecular markers were not proven influential on survival in our cohort, probably due to the limitation of small sample size. Thirdly, the reasons for delayed initiation of CRT were not all collected in our research. For example, among the 18 patients who started CRT beyond 6 weeks in our study, as many as 10 patients were from rural areas. These patients from rural areas started CRT late because they didn't return to the hospital timely after surgery due to their poor disease cognition. When disease of these patients progressed after standard CRT, delay to seek treatment might still happen again, thus led to poor survival. Besides, low education, poor economic conditions and poor recovery from surgery might also lead to delay of initiation of CRT, which was indicated in our cohort. In our future studies, we would collect more detailed clinical, social and economic data might be associated with delay of CRT and explore which of the delays could be avoided, thus improving the survival outcomes of GBM patients.

\section{Conclusions}

Our data suggested initiation of postoperative CRT longer than 6 weeks was associated with worsened survival in GBM patients. Prolonged delay of CRT could lead to early tumor regrowth and reduce the survival improvement brought by maximal surgical resection. Thus we suggested CRT should be initiated within 6 weeks after surgery.

\section{Acknowledgments}

Funding: None.

\section{Footnote}

Reporting Checklist: The authors have completed the STROBE reporting checklist. Available at http://dx.doi. org/10.21037/tcr-20-1718

Data Sharing Statement: Available at http://dx.doi. org/10.21037/tcr-20-1718

Conflicts of Interest: All authors have completed the ICMJE uniform disclosure form (available at http://dx.doi. org/10.21037/tcr-20-1718). The authors have no conflicts of interest to declare.

Etbical Statement: The authors are accountable for all aspects of the work in ensuring that questions related to the accuracy or integrity of any part of the work are appropriately investigated and resolved. The study was conducted in accordance with the Declaration of Helsinki (as revised in 2013). This study was approved by the Ethics Committee of Ruijin Hospital affiliated to Shanghai Jiao Tong University School of Medicine (ID: 2020-192), and written informed consent was not required because of the retrospective nature of the study.

Open Access Statement: This is an Open Access article distributed in accordance with the Creative Commons Attribution-NonCommercial-NoDerivs 4.0 International License (CC BY-NC-ND 4.0), which permits the noncommercial replication and distribution of the article with the strict proviso that no changes or edits are made and the original work is properly cited (including links to both the formal publication through the relevant DOI and the license). See: https://creativecommons.org/licenses/by-nc-nd/4.0/.

\section{References}

1. Stupp R, Mason WP, van den Bent MJ, et al. Radiotherapy plus concomitant and adjuvant temozolomide for glioblastoma. N Engl J Med 2005;352:987-96.

2. Van Meir EG, Hadjipanayis CG, Norden AD, et al. Exciting new advances in neuro-oncology: the avenue to a cure for malignant glioma. CA Cancer J Clin 2010;60:166-93.

3. Stupp R, Hegi ME, Mason WP, et al. Effects of radiotherapy with concomitant and adjuvant temozolomide versus radiotherapy alone on survival in glioblastoma 
in a randomised phase III study: 5-year analysis of the EORTC-NCIC trial. Lancet Oncol 2009;10:459-66.

4. Richards MA, Westcombe AM, Love SB, et al. Influence of delay on survival in patients with breast cancer: a systematic review. Lancet 1999;353:1119-26.

5. Buchholz TA, Austin-Seymour MM, Moe RE, et al. Effect of delay in radiation in the combined modality treatment of breast cancer. Int J Radiat Oncol Biol Phys 1993;26:23-35.

6. Chen Z, King W, Pearcey R, et al. The relationship between waiting time for radiotherapy and clinical outcomes: a systematic review of the literature. Radiother Oncol 2008;87:3-16.

7. Barton MB, Morgan G, Smee R, et al. Does waiting time affect the outcome of larynx cancer treated by radiotherapy? Radiother Oncol 1997;44:137-41.

8. Choi N, Baumann M, Flentjie M, et al. Predictive factors in radiotherapy for non-small cell lung cancer: present status. Lung Cancer 2001;31:43-56.

9. Wyatt RM, Jones BJ, Dale RG. Radiotherapy treatment delays and their influence on tumour control achieved by various fractionation schedules. Br J Radiol 2008;81:549-63.

10. Burnet NG, Jena R, Jefferies SJ, et al. Mathematical modelling of survival of glioblastoma patients suggests a role for radiotherapy dose escalation and predicts poorer outcome after delay to start treatment. Clin Oncol (R Coll Radiol) 2006;18:93-103.

11. Gerlee P, Nelander S. The impact of phenotypic switching on glioblastoma growth and invasion. PLoS Comput Biol 2012;8:e1002556.

12. Wang CH, Rockhill JK, Mrugala M, et al. Prognostic significance of growth kinetics in newly diagnosed glioblastomas revealed by combining serial imaging with a novel biomathematical model. Cancer Res 2009;69:9133-40.

13. Stensjøen AL, Solheim O, Kvistad KA, et al. Growth dynamics of untreated glioblastomas in vivo. Neuro Oncol 2015;17:1402-11.

14. De Barros A, Attal J, Roques M, et al. Impact on survival of early tumor growth between surgery and radiotherapy in patients with de novo glioblastoma. J Neurooncol 2019;142:489-97.

15. Villanueva-Meyer JE, Han SJ, Cha S, et al. Early tumor growth between initial resection and radiotherapy of glioblastoma: incidence and impact on clinical outcomes. J Neurooncol 2017;134:213-9.

16. Wee CW, Kim E, Kim TM, et al. Impact of interim progression during the surgery-to-radiotherapy interval and its predictors in glioblastoma treated with temozolomide-based radiochemotherapy. J Neurooncol 2017;134:169-75.

17. Delgado-López PD, Corrales-Garcia EM. Survival in glioblastoma: a review on the impact of treatment modalities. Clin Transl Oncol 2016;18:1062-71.

18. Lacroix M, Abi-Said D, Fourney DR, et al. A multivariate analysis of 416 patients with glioblastoma multiforme: prognosis, extent of resection, and survival. J Neurosurg 2001;95:190-8.

19. Laws ER, Parney IF, Huang W, et al. Survival following surgery and prognostic factors for recently diagnosed malignant glioma: data from the Glioma Outcomes Project. J Neurosurg 2003;99:467-73.

20. Adamson C, Kanu OO, Mehta AI, et al. Glioblastoma multiforme: a review of where we have been and where we are going. Expert Opin Investig Drugs 2009;18:1061-83.

21. Qin JJ, Liu ZX, Wang JM, et al. Prognostic factors influencing clinical outcomes of malignant glioblastoma multiforme: clinical, immunophenotypic, and fluorescence in situ hybridization findings for $1 \mathrm{p} 19 \mathrm{q}$ in 816 chinese cases. Asian Pac J Cancer Prev 2015;16:971-7.

22. Valduvieco I, Verger E, Bruna J, et al. Impact of radiotherapy delay on survival in glioblastoma. Clin Transl Oncol 2013;15:278-82.

23. Seidlitz A, Siepmann T, Lock S, et al. Impact of waiting time after surgery and overall time of postoperative radiochemotherapy on treatment outcome in glioblastoma multiforme. Radiat Oncol 2015;10:172.

24. Nathan JK, Brezzell AL, Kim MM, et al. Early initiation of chemoradiation following index craniotomy is associated with decreased survival in high-grade glioma. J Neurooncol 2017;135:325-33.

25. Sun MZ, Oh T, Ivan ME, et al. Survival impact of time to initiation of chemoradiotherapy after resection of newly diagnosed glioblastoma. J Neurosurg 2015;122:1144-50.

26. Graus F, Bruna J, Pardo J, et al. Patterns of care and outcome for patients with glioblastoma diagnosed during 2008-2010 in Spain. Neuro Oncol 2013;15:797-805.

27. Do V, Gebski V, Barton MB. The effect of waiting for radiotherapy for grade III/IV gliomas. Radiother Oncol 2000;57:131-6.

28. Irwin C, Hunn M, Purdie G, et al. Delay in radiotherapy shortens survival in patients with high grade glioma. J Neurooncol 2007;85:339-43.

29. Spratt DE, Folkert M, Zumsteg ZS, et al. Temporal relationship of post-operative radiotherapy with temozolomide and oncologic outcome for glioblastoma. J 
Neurooncol 2014;116:357-63.

30. Pollom EL, Fujimoto DK, Han SS, et al. Newly diagnosed glioblastoma: adverse socioeconomic factors correlate with delay in radiotherapy initiation and worse overall survival. J Radiat Res 2018;59:i11-i18.

31. Loureiro LV, Victor Eda S, Callegaro-Filho D, et al. Minimizing the uncertainties regarding the effects of delaying radiotherapy for Glioblastoma: A systematic review and meta-analysis. Radiother Oncol 2016;118:1-8.

32. Blumenthal DT, Won M, Mehta MP, et al. Short delay in initiation of radiotherapy may not affect outcome of patients with glioblastoma: a secondary analysis from the radiation therapy oncology group database. J Clin Oncol 2009;27:733-9.

33. Blumenthal DT, Won M, Mehta MP, et al. Short delay in initiation of radiotherapy for patients with glioblastomaeffect of concurrent chemotherapy: a secondary analysis from the NRG Oncology/Radiation Therapy Oncology Group database. Neuro Oncol 2018;20:966-74.

34. Wang TJ, Jani A, Estrada JP, et al. Timing of Adjuvant Radiotherapy in Glioblastoma Patients: A SingleInstitution Experience With More Than 400 Patients. Neurosurgery 2016;78:676-82.

Cite this article as: Zhang M, Xu F, Ni W, Qi W, Cao W, Xu C, Chen J, Gao Y. Survival impact of delaying postoperative chemoradiotherapy in newly-diagnosed glioblastoma patients. Transl Cancer Res 2020;9(9):5450-5458. doi: 10.21037/tcr-20-1718
35. Champ CE, Siglin J, Mishra MV, et al. Evaluating changes in radiation treatment volumes from post-operative to same-day planning MRI in High-grade gliomas. Radiat Oncol 2012;7:220.

36. Peker S, Abacioglu U, Sun I, et al. Irradiation after surgically induced brain injury in the rat: timing in relation to severity of radiation damage. J Neurooncol 2004;70:17-21.

37. Azizi AA, Paur S, Kaider A, et al. Does the interval from tumour surgery to radiotherapy influence survival in paediatric high grade glioma? Strahlenther Onkol 2018;194:552-9.

38. Thomson CL, Watterson A, Pritchard C, et al. Effects of Waiting Times and Adjuvant Chemotherapy in Patients with Glioblastoma Multiforme. Clin Oncol 2007;19:47-8.

39. Lai R, Hershman DL, Doan T, et al. The timing of cranial radiation in elderly patients with newly diagnosed glioblastoma multiforme. Neuro Oncol 2010;12:190-8.

40. Noel G, Huchet A, Feuvret L, et al. Waiting times before initiation of radiotherapy might not affect outcomes for patients with glioblastoma: a French retrospective analysis of patients treated in the era of concomitant temozolomide and radiotherapy. J Neurooncol 2012;109:167-75. 